\title{
La Surveillance Prénatale, Au Maroc Cas Des Femmes Ayant Accouché À La Maternité Souissi, CHU (Ibn Sina) Rabat
}

\author{
Y. El Housni \\ E.Boussalwa \\ Laboratoire de Biodiversité et Ressources Naturelles, \\ Université Ibn Tofail, Kenitra, Maroc
}

\section{A.Kharbach}

Chef de Service de Gynécologie Obstétrique et d'Endocrinologie à la Maternité Souissi, CHU Rabat, (Université Mohamed V) Maroc

\section{A. Khadmaoui}

Laboratoire de Biométrie et Génétique de la Population, Université Ibn Tofail, Kenitra, Maroc

doi: 10.19044/esj.2016.v12n6p232 URL:http://dx.doi.org/10.19044/esj.2016.v12n6p232

\begin{abstract}
The aim of this study is to analyse the prenatal care performed to the women who gave birth at the Souissi Maternity, Ibn Sina University Hospital of Rabat, Morocco. This study is based on a historical cohort with prospective data collection, during the period of 2 April 2013 to 14 April 2013, with 230 patients Hospitalized to deliver. According to the study 96, 5 $\%$ of the women interviewed received at least one prenatal consultation from a qualified staff. Despite the high rate of coverage with antenatal care, it was found that more than half of women did not benefit from the following services: size measuring, urinalysis, rubella and toxoplasmosis serology. We also noted a growing concern among women for pregnancy monitoring quality, since $43 \%$ were simultaneously monitored by several categories of staff. To improve the quality of prenatal care, we recommend that health professionals complete physical and diagnostic tests, also provide more information about continuity of care during prenatal consultations.
\end{abstract}

Keywords: Pregnancy / Prenatal care (PNC)/ Morocco

Résumé

Le but de notre étude est de montrer et analyser la surveillance prénatale réalisée par les femmes ayant accouché à la Maternité Souissi de 
Rabat. Il s’agit d'une cohorte historique avec recueil prospectif des données, durant la période allant du 2 au 14 avril 2013 auprès de 230 patientes hospitalisées en suites de couches. Selon notre étude 96,5 \% des femmes interviewées ont bénéficié au moins d'une consultation prénatale chez un personnel qualifié. Malgré le taux élevé de la couverture de ces consultations, on a trouvé que plus de la moitié des femmes n'ont pas bénéficié des prestations suivantes : la mesure de la taille, l'analyse des urines, la sérologie de la rubéole et la sérologie de la toxoplasmose. On note aussi, une préoccupation croissante chez les femmes d'avoir une surveillance de grossesse de qualité ; puisque $43 \%$ ont été suivies simultanément par différentes catégories de personnel. Pour améliorer la qualité de la consultation prénatale, nous recommandons aux professionnels de santé de compléter l'examen physique et les examens paracliniques et de donner plus d'informations aux gestantes sur l'importance de la continuité de la surveillance prénatale.

Mots-clés : La grossesse, les soins prénatals, Maroc.

\section{Introduction}

Chaque jour au moins 800 femmes environ succombent dans le monde suite à des complications liées à la grossesse et à l'accouchement, soit un décès toutes les 2 minutes ce qui représente 292.000 décès maternels par an (OMS, 2014).

Au Maroc, d'après l'enquête nationale démographique à passages répétés 2009/2010 menée par le Haut-Commissariat au Plan du Maroc a révélé que le taux de mortalité maternelle se situe à 112 décès pour 100000 naissances vivantes soit une réduction de 60\% par rapport à 1990 (Ministère de la santé, 2011).

Ce progrès expliqué, en partie, par l'amélioration des soins prénataux. La proportion des femmes ayant bénéficié des consultations prénatales (CPN) est passé de $32 \%$ en 1992 à $58 \%$ en 1997 puis à $68 \%$ en 2004 pour atteindre 77,1\% en 2011. En milieu rural, cette proportion a gagné 44,7 point entre 1992et 2011(ENPSF, 2012).

La consultation prénatale permet de sensibiliser les gestantes sur les signes d'alerte et de danger, de détecter et de traiter précocement les situations à risque et d'améliorer le recours à l'aide appropriée lors de l'accouchement. Les consultations idéales à 12, 26, 32 et 36 semaines d'aménorrhée ont pour but de :

- dépister trois risques majeurs : la cicatrice utérine, la présentation dystocique et la rupture prématurée des membranes.

- prévenir/ dépister et traiter les complications telles que l'hypertension gravidique, les infections (maladies vénériennes, VIH, 
tétanos, infection urinaire), l'anémie et la carence en micronutriments, le diabète gestationnel.

- fournir des conseils, le soutien et les informations aux parturientes et aux familles sur les signes d'alerte et de danger et la préparation de l'accouchement (Bonono \& Ongolo-Zogo, 2012)

Au Maroc, la consultation prénatale est organisée au niveau des espaces conçus et équipés à cet effet et ce au niveau des structures de soins de santé de base (centres de santé urbains et ruraux, dispensaires), maisons d'accouchement, maternités hospitalières et points de contact des équipes mobiles et elle est assumé par la sage-femme/infirmière, le médecin généraliste et le gynécologue / obstétricien. Les femmes dont la grossesse évolue normalement bénéficient de quatre consultations prénatales et chaque consultation est structurée et comporte un contenu ciblé adapté au stade de la grossesse (Décret $\left.n^{\circ} 2-05-733,2005\right)$. Certaines examens complémentaires (biologiques et radiologiques) sont réalisés systématiquement et d’autres selon l'indication médicale.

Le présent travail a pour but de montrer et analyser la surveillance prénatale réalisée chez les femmes ayant accouché à la Maternité Souissi de Rabat à travers l'analyse des prestations offertes lors des visites prénatales et de déterminer leurs caractéristiques épidémiologiques.

\section{Matériel et méthodes.}

Il s’agit d'une étude descriptive prospective à visée quasi exhaustive menée à la Maternité Souissi de la période allant du 2 au 14 avril 2013 auprès, de 230 patientes hospitalisées en suites de couches au niveau de la Maternité Souissi du CHU de Rabat, Maroc

Etaient inclues dans l'étude toutes les femmes ayant accouché d'un enfant vivant ou mort-né pendant la période d'étude au niveau de la maternité et ayant passé leurs suites de couches au niveau des deux services de l'étage 1 et l'étage 3.

Les femmes pouvant répondre à l'un des critères suivants ont été exclues de l'étude :

- $\quad$ Refus de la patiente à participer à l’étude.

- $\quad$ Accouchement à l'extérieur de la Maternité Souissi.

Les données étaient recueillies au moyen d'un questionnaire papier comportant deux parties : la première concernait les caractéristiques des patientes, la deuxième portait sur le suivi de grossesse et l'accouchement. Les caractéristiques sociodémographiques des mères et le déroulement de la surveillance prénatale étaient renseignés lors d'un entretien avec les femmes avant leur sortie de la maternité. Les informations médicales relatives à la consultation prénatale et aux antécédents étaient collectées à 
partir du carnet de santé et le dossier médical de l’accouchement de la femme.

Une analyse descriptive simple a été réalisée à l'aide du logiciel Excel et SPSS version 20.0. Le test chi-deux $(\chi 2)$ a été utilisé pour déceler les liaisons significatives entres les différentes variables étudiées.

\section{Résultats et discussion}

\section{Profil des femmes enceintes}

Des 230 femmes interviewées, nous avons vérifié que le groupe des 18 à 24 ans représente le plus grand pourcentage de l'échantillon avec 32,2 \% ( $n=74)$. S'ensuit le groupe des 25 à 29 ans avec 23,9\% $(n=55)$, des 30 à 34 ans avec 22,6\% $(n=52)$ et finalement le groupe des 35 à 39 ans avec $16,1 \%(n=37)$. Il est important également de signaler que, dans cet échantillon, le groupe des 16 à 17 ans représente $1,7 \%(n=4)$ et celui de 40 à 44 ans représente $3,5 \%(n=8)$. En ce qui concerne l'état civil, nous avons constaté que 94,78 \% des femmes $(n=218)$ étaient mariées, tout en démontrant que la majorité des nouveau-nés naissent encore au sein de l'institution matrimoniale. On note que les 230 femmes ayant indiqué leur scolarité, 57 (24,78\%) sont des analphabètes. Parmi les 173 femmes ayant suivi une scolarité, 24,34\% s’étaient arrêtées au primaire, 20,86\% au collège, 18,26\% au lycée et 9,56 \% à l’issue d’études supérieures.

\section{Bref « regard » sur l'histoire de la grossesse}

Un pourcentage de $77 \%$ des femmes $(n=177)$ a affirmé que leur grossesse était désirée et $23 \%(n=53)$ ont été surprises par le diagnostic. De ces 53 femmes, 33 ont été tombées enceintes suite à une mauvaise utilisation de la contraception. Quant au nombre de grossesses, 44,8 \% $(n=103)$ des femmes interviewées ont affirmé être à leur première grossesse, 21,7 \% $(n=50)$ à leur seconde, 20,4 \% $(n=47)$ à leur troisième et seulement $13 \%(n=30)$ à leur quatrième, cinquième sixième ou septième grossesse. Il faut signaler que 14.4 \% des interviewées ont déclaré avoir déjà eu un avortement lors de grossesses précédentes et 1,7 \% un enfant mort-né. Ces situations ont influencé la présente grossesse, qui a été vécue avec plus d'anxiété et de grandes peurs. De cette façon, ces femmes ont été plus utilisatrices des consultations prénatales et ont été plus réceptives aux conseils des professionnels de santé.

\section{Caractéristiques de la surveillance prénatale au Maroc selon l'enquête nationale sur la population et la santé familiale (ENPSF-2011).}

$\mathrm{Au}$ Maroc, toutes les femmes enceintes ont un accès gratuit aux soins prénataux. La proportion des femmes qui ont bénéficié de consultations prénatales avec un personnel qualifié (médecin ou infirmier/sage-femme) est 
de l'ordre de 77,1\% (contre 67,8\% en 2004). Cette proportion diffère significativement par milieu (91,1\% en milieu urbain contre 62,7\% en milieu rural). Les naissances du premier rang sont les plus nombreuses à avoir bénéficié d'une consultation prénatale (87,8\% contre 63,9\% pour les naissances du rang quatre et plus).

On constate également qu'environ $99 \%$ des femmes ayant le certificat des études secondaires ont bénéficié des consultations prénatales contre 73,3\% des femmes n'ayant aucun certificat scolaire (ENPSF ,2012).

\section{Surveillance et accompagnement de la grossesse dans notre étude.}

Nous avons constaté que, 97\% les femmes enceintes ont été surveillées pendant la grossesse. Selon les résultats de la figure (1), 21 \% des femmes ont été accompagnées exclusivement par le médecin obstétricien/gynécologue, 22 \% par le médecin généraliste et 14\% seulement par la sage-femme/infirmière et $27 \%$ par le médecin généraliste et la sagefemme/infirmière. De cette façon, environ $37 \%$ des femmes enceintes ont consulté un médecin obstétricien/gynécologue pendant la grossesse et 58 \% un médecin généraliste.

D’après notre étude on constate que la CPN a été médicalisée chez $75.04 \%$ des femmes et d'après le graphe de la figure 1, nous pouvons observer aussi l'existence d'une «surconsommation » de consultations réalisées par $43 \%$ des femmes, dans la mesure où elles ont été suivies simultanément au centre de santé, en cabinet de consultations du généraliste ou du gynécologue /obstétricien. Ce double suivi peut, éventuellement, être expliqué par l'existence de peurs et d'anxiété, par un manque de confiance dans les soins de santé primaires et du médecin généraliste ou même par un excès de préoccupation de la part des parents.
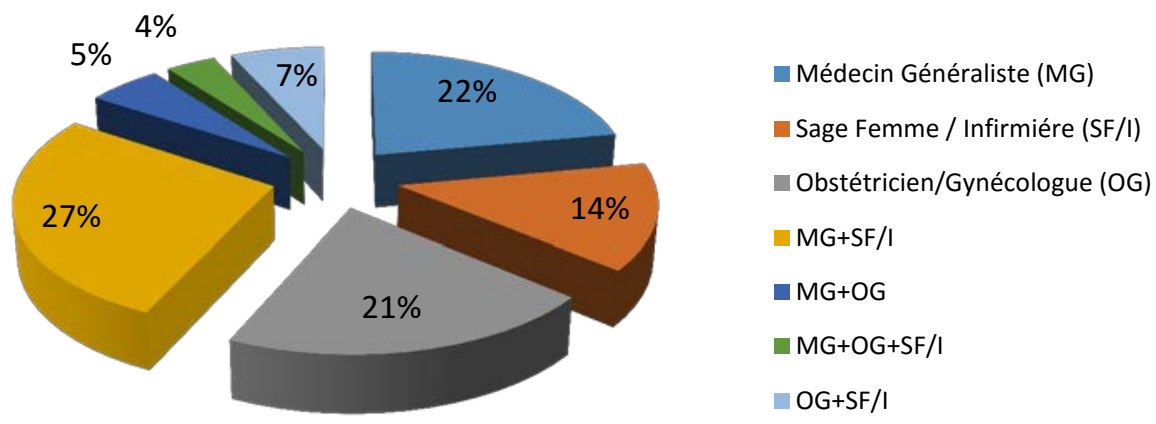

Figure 1 : Répartition des femmes selon le profil du personnel consulté au cours de la grossesse. 
L’option du suivi de la grossesse par un médecin obstétricien, résulte de la perception que celui-ci détient plus de connaissances et, de ce fait, la grossesse sera mieux surveillée. Néanmoins, on observe une relation forte entre le niveau d'instruction de la femme et l'option retenue pour la consultation réalisée par un médecin obstétricien/gynécologue $(\chi 2=36,4$, ddl $=15 ; p=0,002)$ et il y a aussi une relation de dépendance entre le niveau d'instruction du conjoint et le profil du personnel consulté au cours de la grossesse $\left(\chi^{2}=36,74, \mathrm{ddl}=15 ; p=0,001\right)$.

Par contre dans notre échantillon on n'a pas trouvé une influence de l'âge de la femme, de la nature de l'habitat de la femme et la nature de la fonction du conjoint sur le choix du personnel de suivi de la grossesse.

Cependant, quelques femmes ont valorisé le travail réalisé par la sage-femme/infirmière (SF/I) au cours de la grossesse puisque $14 \%$ de ces femmes ont réalisé un suivi exclusif chez elles.

\section{Début de la surveillance prénatale.}

Selon les données de la figure 2 on constate que, quatre femmes sur cinq $(78,82 \%)$, ont effectué la première visite prénatale à un stade précoce de la grossesse, c'est-à-dire dans les 3 premiers mois, Cette proportion est supérieure à celle de l'enquête nationale de 2011 au Maroc (ENPSF, 2012) qui avait trouvé $69,9 \%$. Par contre $16.67 \%$ des femmes ont effectué leur première visite entre le quatrième et le cinquième mois de grossesse et pour $4,05 \%$ des naissances, la première visite prénatale n’a été effectuée qu'à partir de 6 mois de grossesse. Dans seulement 0,46 \% des cas, les femmes ont attendu au moins le huitième mois pour effectuer la première visite.

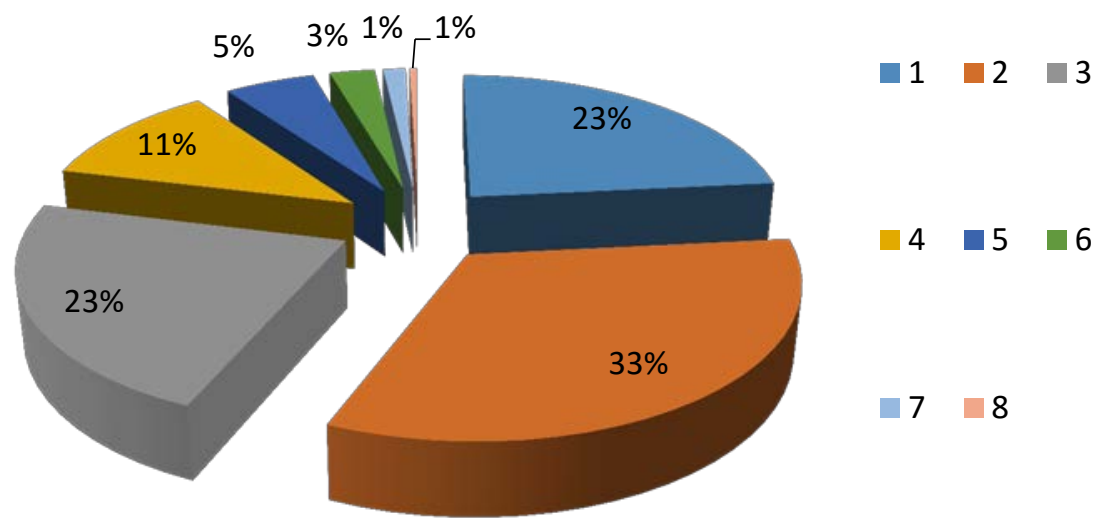

Figure 2 : La répartition des femmes selon l’âge de la première CPN en mois. 


\section{Nombre de consultations réalisées}

Pour être efficace, les soins prénatals doivent être effectués à un stade précoce de la grossesse et, surtout, ils doivent se poursuivre avec une certaine régularité jusqu'à l'accouchement. L'Organisation Mondiale de la Santé (OMS) recommande, au moins, quatre visites prénatales, à intervalles réguliers tout au long de la grossesse. Selon la figure 3 sur le nombre de visites prénatales effectuées, près des deux tiers des naissances $(65,76 \%)$ ont fait l'objet de 4 visites prénatales ou plus, ce qui est en accord avec les recommandations de l'OMS. Cette proportion est inférieure à celle de S. El Mhamdi en Tunisie (Mhamdi et al, 2010) qui avait trouvé 82,5\%. Par ailleurs, on note que pour 28,84 \% des naissances, les mères ont effectué 2 à 3 visites et que pour $5,4 \%$ des naissances, elles n'en ont effectué qu'une seule.

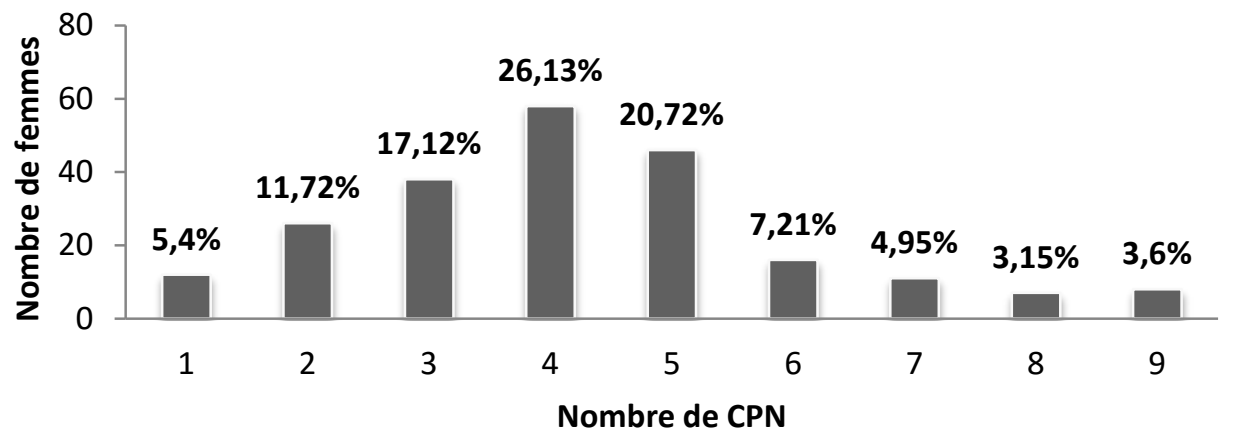

Figure 3 : la répartition des femmes selon le nombre de CPN.

\section{Proportion (en \%) des femmes ayant reçu des soins prénatals qualifiés selon la nature des examens reçus.}

Les examens cliniques et complémentaires représentent une arme efficace pour la détection des pathologies accompagnant la grossesse. Le ministère de la santé a classé les examens en deux : Certaines examens sont réalisés systématiquement et d'autres selon l'indication médicale. (Direction de la population, 2011).

S'agissant des prestations reçues lors des visites prénatales réalisées par les femmes interviewées ; il ressort du graphique 4 que l'examen le plus fréquemment réalisé est la mesure de la tension artérielle (98,6\%). Ce taux est supérieur à celui d'ENSFP-20011 qui avait trouvé 90,6 \%. , suivi par l'échographie obstétricale (97,3\%) (L'ENSFP-2011 avait trouvé 79,4\%) et le groupage $\mathrm{ABO} / \mathrm{Rhésus}(89,6 \%)$ et alors que les examens les moins réalisés sont l'analyse des urines $(17,6 \%)$; cette faible demande de recherche d'albumine et de sucre dans les urines est difficile à expliquer alors que son coût n'est pas élevé. L'examen des membres inférieurs (35,6\%) et la mesure 
de la taille (40,1\%). Le faible taux de réalisations de quelques examens peut être expliqué :

- soit par la méconnaissance du personnel de l’importance de ces examens dans la surveillance prénatale.

- soit par le fait que le personnel de santé n’est pas au courant des nouvelles mises à jour du programme de surveillance de la grossesse.

- soit par les difficultés financières qui empêchent certaines femmes à réaliser certains examens complémentaires qui ne sont pas disponibles dans les structures de santé.

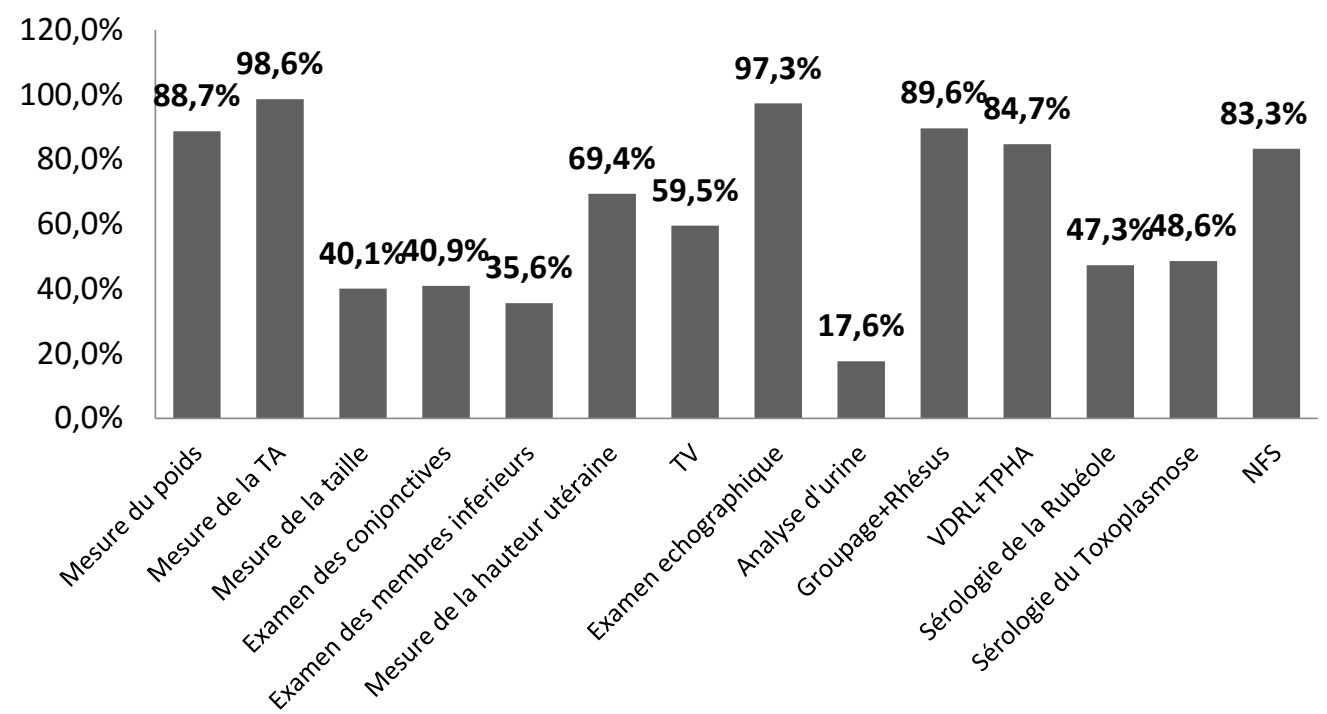

La figure 4 : proportion (en \%) des femmes ayant reçu des soins prénatals qualifiés selon la nature des examens reçus.

\section{Conclusion}

Au Maroc, l’évaluation continue des programmes de santé démontre une amélioration de la santé maternelle. Bien que les mesures introduites ces dernières années entrainent une baisse remarquable des valeurs de la mortalité maternelle et périnatale (Ministère de la santé, 2012).

D’après notre étude on note que certaines femmes ont eu recours à plusieurs catégories de personnel pour la surveillance de leurs grossesses en vue d'avoir une meilleure qualité des soins. Nous avons trouvé aussi que le nombre de consultations réalisées et la précocité de la surveillance prénatale sont suffisants. Par contre, plus de la moitié des femmes n’ont pas bénéficié des prestations suivantes : la mesure de la taille, l'analyse des urines ; la sérologie de la rubéole et la sérologie de la toxoplasmose. 
De ce fait, pour améliorer la qualité de la consultation prénatale ; nous recommandons aux professionnels de santé de connaître et valoriser certains facteurs associés aux caractéristiques de la femme et de compléter l'examen physique et les examens paracliniques et de donner plus d'informations aux gestantes sur l'importance de la continuité des soins au cours des consultations prénatales.

\section{References:}

Bonono R.C., Ongolo-Zogo. Optimiser l'utilisation de la consultation prénatale au Cameroun. Centre pour le Développement des Bonnes Pratiques en Santé - Hôpital Central. Yaoundé, Cameroun, 2012.

Décret $n^{\circ} 2-05-733$ du 11 joumada II 1426 pris pour l'application de la loi $n^{\circ}$ 65-00 portant code de la couverture médicale de base,2005 .

Direction de la population, Royaume du Maroc, Ministère de la santé. Surveillance de la grossesse et du post-partum, manuel à l'usage des professionnels de santé. Edition Décembre 2011.

ENPSF-2011 : Enquête Nationale sur la Population et la Santé Familiale. Ministère de la Santé DPRF/DPE/SEIS Rabat, Maroc, Décembre 2012.

Mhamdi S-EL, Soltani M-S, Haddad A, Letaief M et Ben Salem K . Les nouveaux critères et la qualité des services de soins de santé dans le gouvernorat de Monastir (Tunisie), La Revue de Santé de la Méditerranée orientale, 16(1):107-112,2010.

Ministère de la santé, Royaume du Maroc. Réduire la mortalité maternelle au Maroc : partager l'expérience et soutenir le progrès, 2011.

Ministère de la santé, Royaume du Maroc. Plan d'action 2012 - 2016 pour accélérer la réduction de la mortalité maternelle et néonatale, 2012.

O.M.S : organisation Mondiale de la Santé. Statistiques sanitaires mondiales 2014. apps.who.int 